\title{
Retinoblastoma outcome at a single institution in South Africa
}

\author{
M Kruger, ${ }^{1} \mathrm{MD}$, PhD; D Reynders, ${ }^{2} \mathrm{MD} ; \mathrm{F}$ Omar, ${ }^{2} \mathrm{MD} ; \mathrm{J}$ Schoeman, ${ }^{3} \mathrm{MSc} ; \mathbf{O}$ Wedi ${ }^{4} \mathrm{MD} ; \mathrm{J}$ Harvey, ${ }^{5} \mathrm{PhD}$ \\ ${ }^{1}$ Department of Paediatrics and Child Health, Faculty of Medicine and Health Sciences, Stellenbosch University, Tygerberg, \\ Cape Town, South Africa \\ ${ }^{2}$ Department of Paediatrics, Faculty of Health Sciences, University of Pretoria, South Africa \\ ${ }^{3}$ Department of Dietetics, Steve Biko Academic Hospital, Pretoria, South Africa \\ ${ }^{4}$ Department of Paediatrics, Faculty of Health Sciences, University of Limpopo, South Africa \\ ${ }^{5}$ Centre for Statistical Consultation, Faculty of Economic and Management Sciences, Stellenbosch University, Western Cape, South Africa
}

Corresponding author: $M$ Kruger (marianakruger@sun.ac.za)

Introduction. Retinoblastoma $(\mathrm{RB})$ is the most common eye cancer in children. Early detection is necessary for cure.

Objective. To compare stage and outcome of children with RB treated at Kalafong Hospital, Pretoria, South Africa (SA), during two time periods (1993 - 2000 and 2001 - 2008, after outreach interventions in 2000 and introduction of compulsory community service for doctors in 1998).

Methods. Data collected included demography (age, gender, date of birth), stage and treatment received. The main outcome measure was disease-free survival and the study end-point was 60 months after diagnosis.

Results. There were 51 patients during the time period 1993 - 2000 (group 1) and 73 during 2001 - 2008 (group 2), with median ages of 32 and 26 months, respectively (marginally significantly younger in group $2 ; p=0.046$ ). In group 1 , the majority (57\%) presented with advanced disease (stages III and IV), with a decline in this proportion in group $2(40 \%)$ indicating a downward but not significant trend $(p=0.075)$. Bilateral disease was diagnosed in $22 \%$ of patients in group 1 and $33 \%$ in group 2 . Overall survival was $33 \%$ and $43 \%$ for groups 1 and 2, respectively. Excluding absconding patients, event-free survival was 50\% in group 1, improving to $68 \%$ in group 2 (not statistically significant; $p=0.18$ ). Fewer patients needed radiotherapy during the second period (statistically significant; $p=0.04$ ), probably because of less advanced disease.

Conclusion. Poor outcome is probably a result of late diagnosis. It is important to implement a strategy that will ensure early diagnosis and optimal management of RB in SA.

S Afr Med J 2014;104(12):859-863. DOI:10.7196/SAMJ.8255

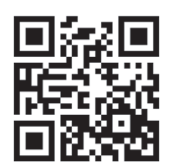

Retinoblastoma $(\mathrm{RB})$ is the most common eye cancer in children aged $<15$ years, accounting for $3 \%$ of all cancers in children. ${ }^{[1]}$ Diagnosis is usually made at a young age ( $<2$ years). The tumour presents unilaterally in the majority of patients $(70-75 \%){ }^{[1]}$ The majority of bilateral tumours (75\%) and about $15 \%$ of unilateral tumours are hereditary, and these patients usually present with more than one tumour in one or both eyes. ${ }^{[2]} \mathrm{RB}$ appears to be more common in Africa and Latin America. ${ }^{[3,4]}$ Survival is excellent in developed countries, where the survival rate is $>95 \%$, but it is only $\sim 50 \%$ in developing countries, mostly because of late diagnosis and extraocular disease at diagnosis. ${ }^{[1,4-6]}$

Late diagnosis of childhood cancer is common in developing countries, and it is important to document the outcome of children with advanced $\mathrm{RB}$ who were treated according to standard treatment protocols. ${ }^{[4,5]}$ In South Africa (SA), several public health interventions have been introduced in the past 20 years, particularly to improve the healthcare of children. Of note is the free healthcare for children under 6 years of age introduced in 1994 and community service for doctors since 1998 in all regions of the country, including rural hospitals with human resource shortages..$^{[7-9]}$

The National Department of Health $(\mathrm{NDoH})$ and the Childhood Cancer Foundation (CHOC), acting in collaboration, introduced an awareness programme with posters depicting the danger signs of childhood cancer in primary healthcare clinics in 2000. ${ }^{[10]}$ Doctors from the universities of the Witwatersrand and Pretoria also undertook outreach visits to referral hospitals in 2001 - 2004 to train staff in early detection of childhood cancer, and especially to check for the absence of a red reflex in the eye in order to detect RB early. Parents were encouraged to note absent red reflex of an eye in photos taken with a flashlight. A satellite paediatric oncology unit (POU) of the POU at Kalafong Hospital, Pretoria, was established in Polokwane, Limpopo Province, in 2007 to assist with early diagnosis, treatment, and referral (only if necessary) as part of improving cancer care for children.

The aim of this research was to compare stage and outcome of children with RB during two time periods, namely 1993 - 2000, before the outreach interventions, and 2001 - 2008, after the outreach interventions.

\section{Patients and methods \\ Setting}

Kalafong Hospital, a large regional hospital, housed the POU before it was moved to Steve Biko Academic Hospital, Pretoria, in December 2009. The POU was established at Kalafong in 1993 and served all children with cancer in the Northern Gauteng region and the provinces of Mpumalanga and Limpopo who were enrolled on prospective treatment protocols between 1993 and 2008.

\section{Design}

We prospectively documented the following data for all patients treated for RB between 1993 and 2008: demographics (date of birth, age, gender and place of birth), medical history, clinical findings, diagnostic procedures, treatment protocol and referral pathway. 


\section{Staging}

The diagnostic procedures for all patients included eye examination under general anaesthesia, a computed tomography scan of the eyes and skull, bone scintigraphy (except in cases of intraocular disease), lumbar puncture for cerebrospinal fluid cell examination and count, and bone marrow aspiration. The patients were staged according to the Grabowski-Abramson classification and the Ellsworth Clinic pathological classification, as follows: ${ }^{[11]}$

- Stage I. Intraocular disease - amenable to local therapy or eye enucleated

- Stage II. Orbital disease (IIa orbital involvement only; IIb post-laminar optic nerve involvement as well) - eye enucleated, microscopic residual tumour, tumour in excision line of the optic nerve

- Stage III. Central nervous system involvement - regional extension and central nervous system metastasis

- Stage IV. Haematogenous metastatic disease

- Stage V. Bilateral disease with or without metastasis.

\section{Treatment outline}

Patients with stage I disease received either local therapy (cryotherapy or brachytherapy) or enucleation, depending on the size of the intraocular tumours and whether it was possible to salvage vision. All patients except those with intraocular disease (stage I) received neoadjuvant chemotherapy. Enucleation or exenteration was performed after two cycles of chemotherapy. Patients with advanced disease (stages III and IV) received either cranial-spinal radiotherapy (external beam radiotherapy) after six cycles of chemotherapy had been completed or iodine-125 brachytherapy in Cape Town. ${ }^{[12,13]}$

The first 12 patients received treatment according to treatment protocol 1 (1993 1994) with the following drugs: ifosfamide $2000 \mathrm{mg} / \mathrm{m}^{2}$, carboplatin $550 \mathrm{mg} / \mathrm{m}^{2}$ and etoposide $150 \mathrm{mg} / \mathrm{m}^{2}$ every 4 weeks for six cycles. Patients with stages II - IV disease also received intrathecal therapy, consisting of methotrexate, cytarabine and Solu-Cortef weekly for 6 weeks. Owing to associated severe haematological toxicity (World Health Organization grade 4), the treatment was changed to treatment protocol 2 (1995 - 2008) with the following drugs: vincristine $1.5 \mathrm{mg} /$ $\mathrm{m}^{2}$, etoposide $300 \mathrm{mg} / \mathrm{m}^{2}$ and carboplatin $550 \mathrm{mg} / \mathrm{m}^{2}$ every 4 weeks for six cycles with intrathecal therapy weekly for 6 weeks.

\section{Patient groups}

The patients were assigned to two groups for two time periods, defined as group 1
Table 1. Demography and management of patients

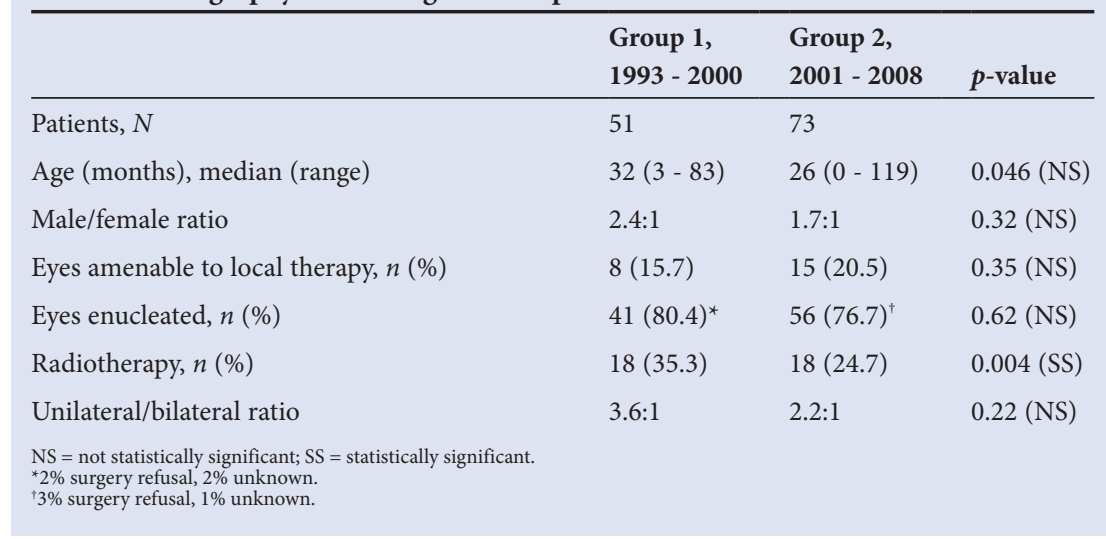

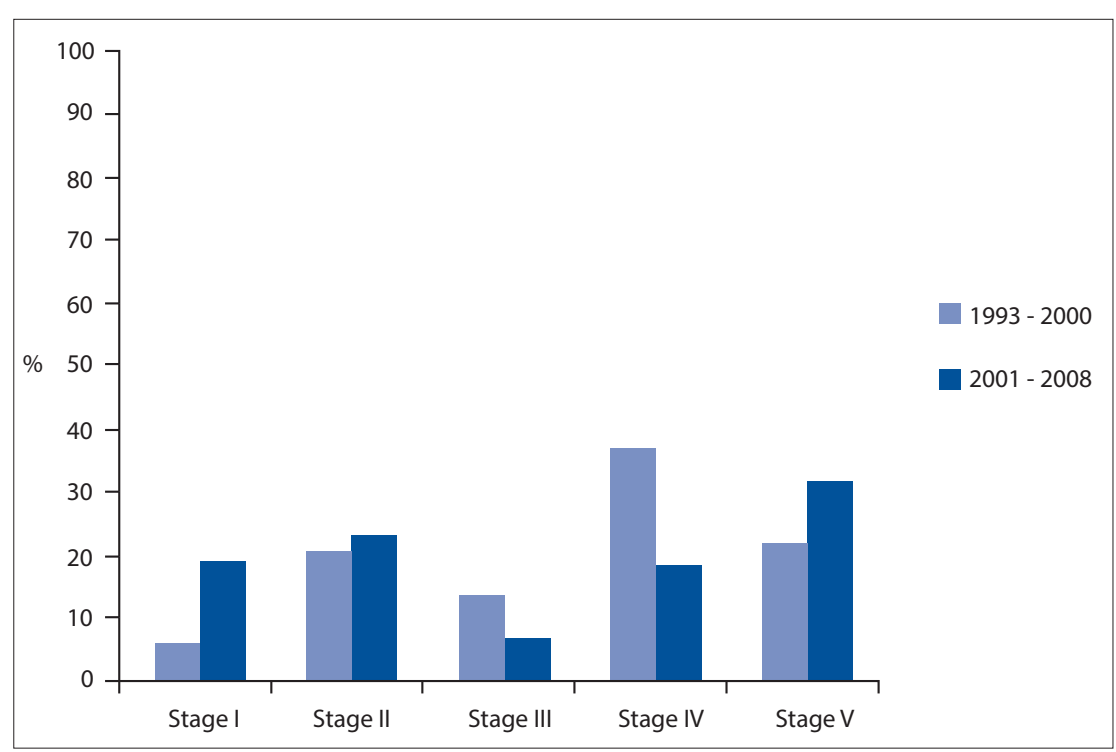

Fig. 1. Stage at diagnosis of $R B$ for the two study groups. ( $R B=$ retinoblastoma; group 1: 1993 - 2000; group 2: 2001 - 2008.)

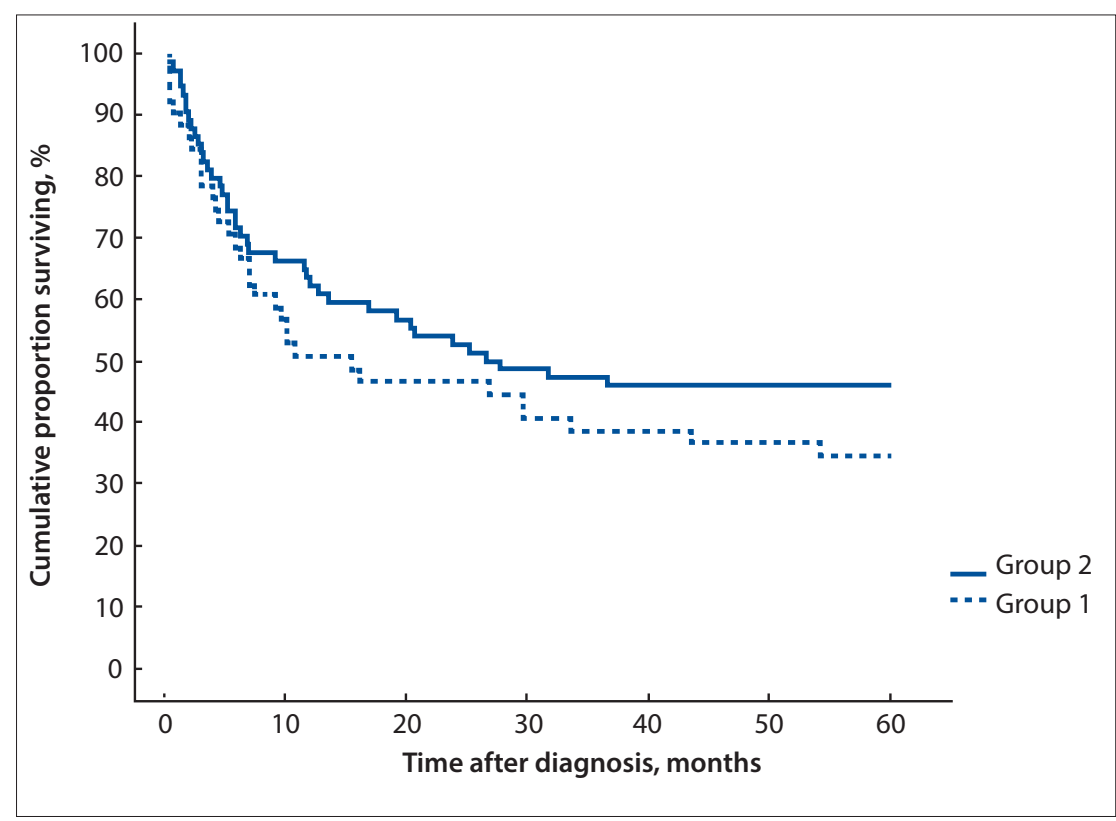

Fig. 2. Overall survival for the two study groups, including absconding patients. (Group 1: 1993 - 2000; group 2: 2001 - 2008.) 


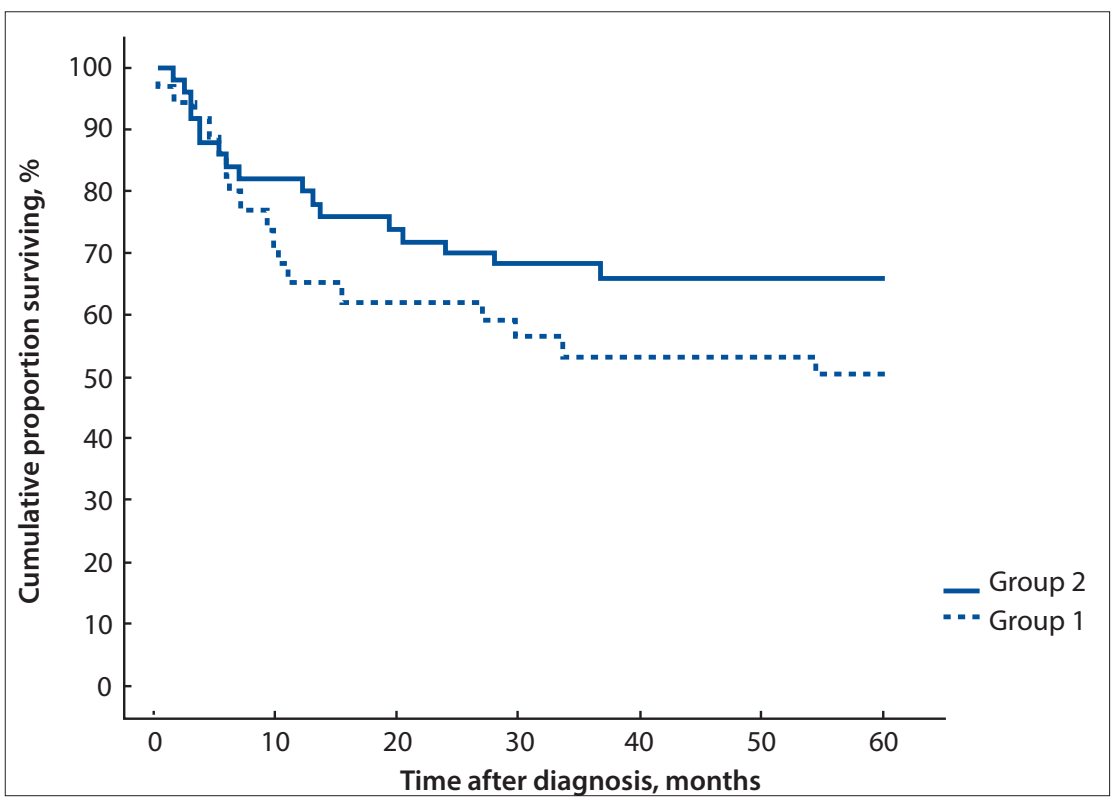

Fig. 3. Overall survival for the two study groups, excluding absconding patients. (Group 1: 1993 - 2000 group 2: 2001 - 2008.)

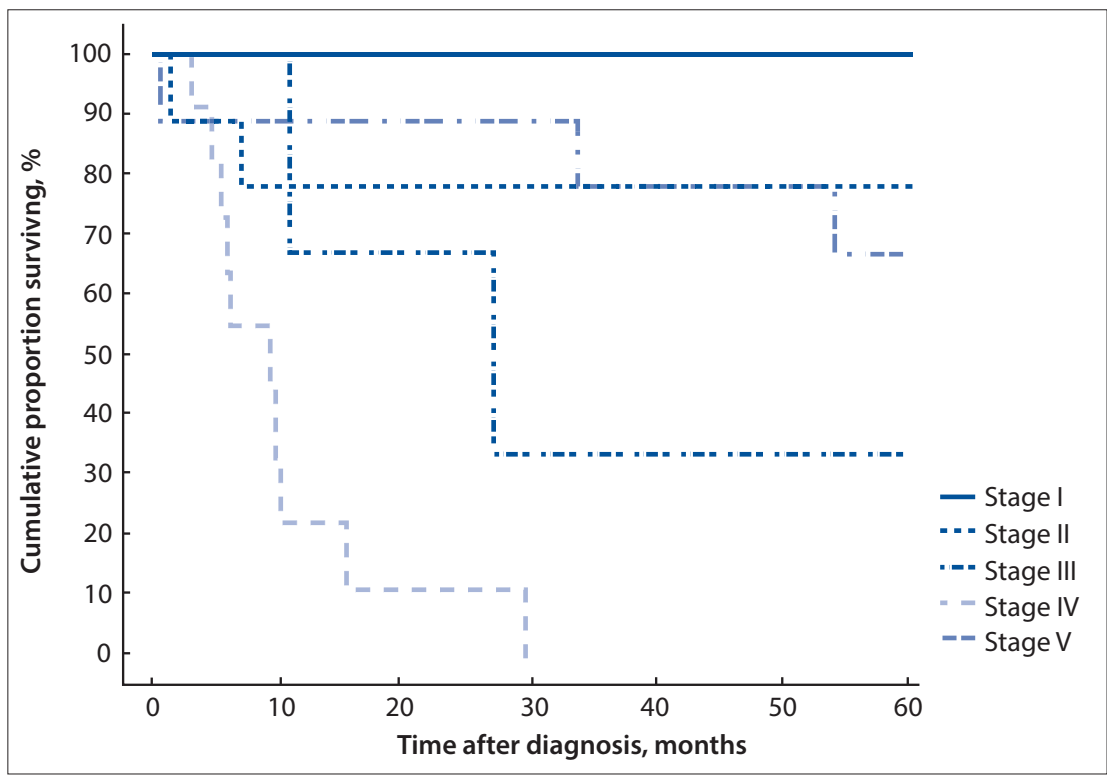

Fig. 4. Survival according to stage at diagnosis for group 1, excluding absconding patients (1993 - 2000).

for the time period 1993 - 2000, before the outreach interventions, and group 2 for the time period 2001 - 2008, during and after the outreach interventions. The second period followed several educational outreach activities to referral centres by staff from the universities of the Witwatersrand and Pretoria, as well as interventions undertaken to improve early diagnosis of childhood cancer by the joint $\mathrm{CHOC}$ and $\mathrm{NDoH}$ awareness campaign. ${ }^{[10]}$ The joint South African Children Cancer Study Group and NDoH awareness campaign distributed posters depicting the danger signs of childhood cancer to all primary care clinics, district
Pearson's $\chi^{2}$ test was used to determine whether differences were significant or not. Kaplan-Meier plots were used to calculate overall survival, and log-rank tests to compare survival rates for different subgroups of patients. A significance level of $p<0.05$ was applied throughout.

\section{Ethics}

The Research Ethics Committee, Faculty of Health Sciences, University of Pretoria, approved this retrospective review of patient records with a waiver of informed consent.

\section{Results}

One hundred and twenty-four patients (51 in group 1 and 73 in group 2) were included in the final data analysis (Table 1). Ten patients were excluded owing to incomplete data (4 patients), transferral to another treatment facility (4 patients) and absconding within an hour from the POU (2 patients).

The median age was 35 months for the combined patient population with unilateral disease and 21 months for patients with bilateral disease (Table 1). The median age was 32 months for group 1 and 26 months for group 2, the second group being slightly younger, with a difference that was marginally statistically significant ( $p=0.046)$. Eleven patients $(22 \%)$ in group 1 and 23 (33\%) in group 2 had bilateral disease. The majority (57\%) in group 1 had advanced disease (29/51 patients: 19 had stage IV disease, 7 had stage III disease and 3 had metastatic bilateral disease (stage V)). There was a downward but not yet statistically significant trend (40\%) in group $2(29 / 73$ patients: 14 had stage IV disease, 5 had stage III disease and 10 had metastatic bilateral disease) ( $p=0.075)$ (Fig. 1). More boys than girls were affected, although the difference was not statistically significant (male/female ratio 2.4:1 for group 1 and 1.7:1 for group 2) (Table 1).

Only 8 eyes were amenable to local therapy in group 1 v. 15 eyes in group 2 (Table 1). Thirty-eight eyes were enucleated and 3 eyes were exenterated in group 1 v. 52 eyes enucleated and 3 eyes exenterated (1 patient with bilateral disease) in group 2 . The parents of three patients refused surgery (1 in group 1, 2 in group 2), and type of surgery was not documented for one child in each group. Fewer patients received radiotherapy in the second group (18/73 patients in group 2 v. 18/51 in group 1), which was statistically significant $(p=0.004)$. Fourteen patients received external beam radiation in group $1 \mathrm{v}$. only 6 in group 2, while only 4 patients received iodine- 125 brachytherapy in group 


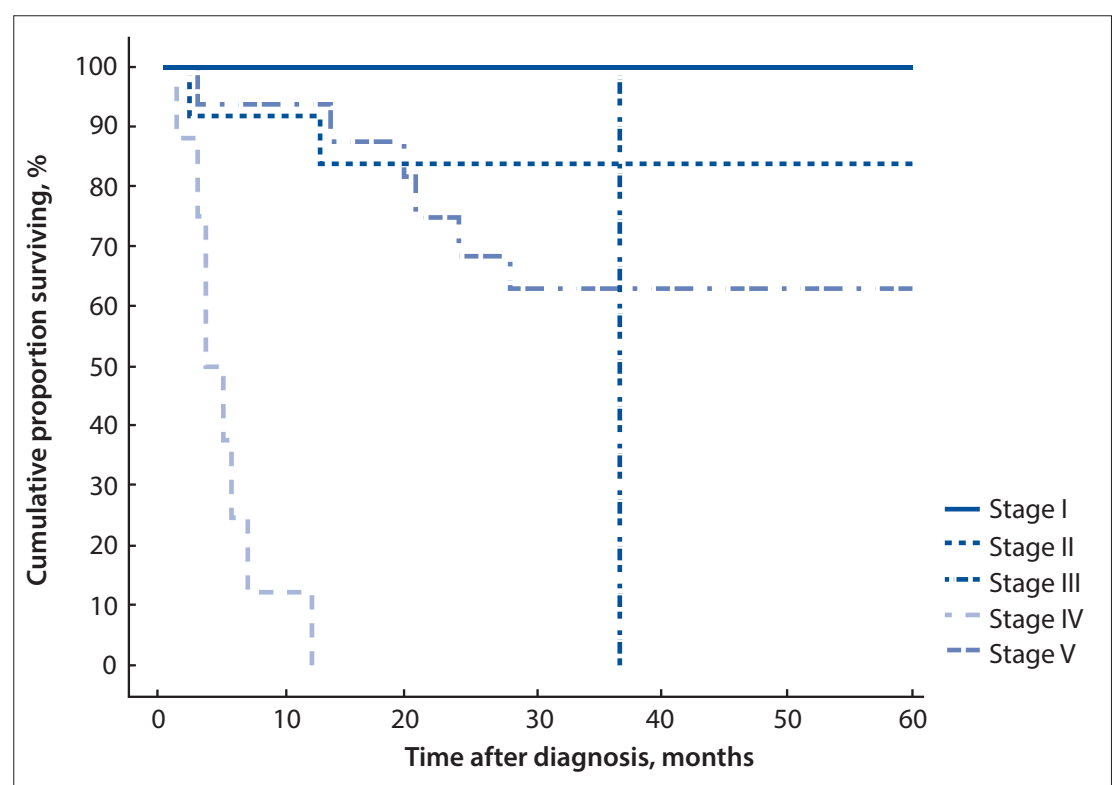

Fig. 5. Survival according to stage at diagnosis for group 2, excluding absconding patients (2001 - 2008).

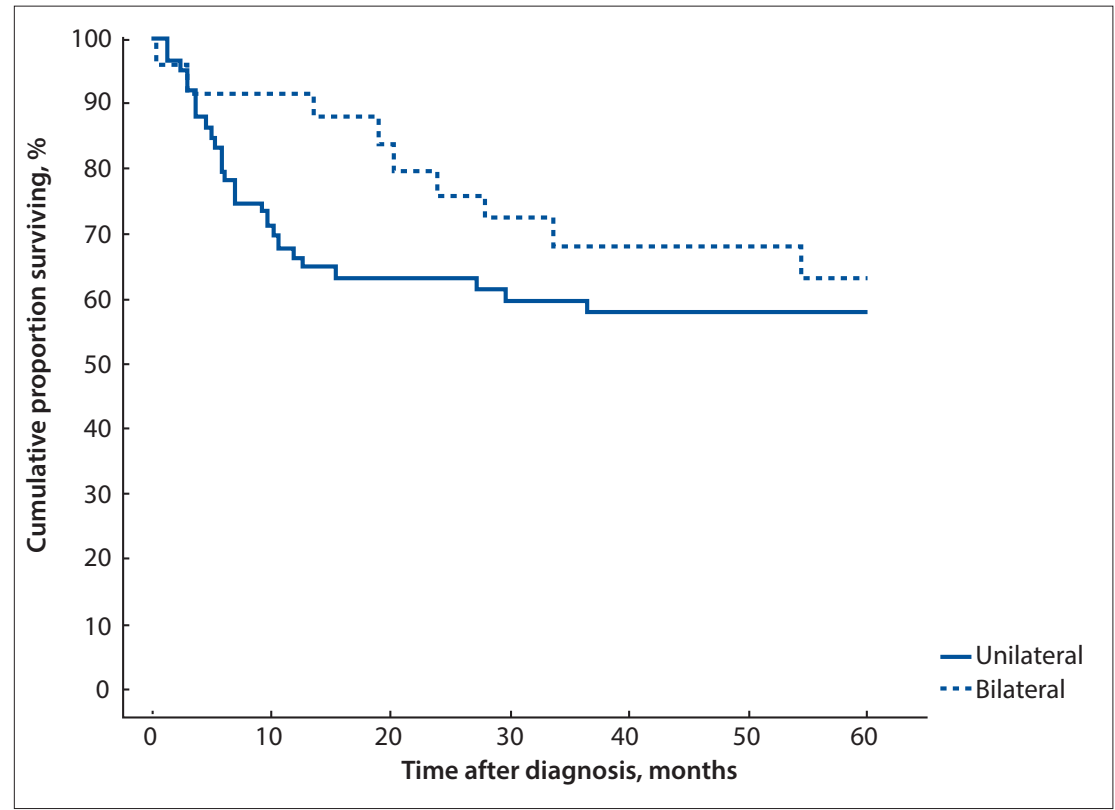

Fig. 6. Overall survival of patients with unilateral v. bilateral disease.

1 , increasing to 12 patients in group 2. Of the patients who received iodine-125 brachytherapy, 2 had stage I disease and 14 bilateral disease. Unilateral disease was more common, as expected, with a ratio of 3.6:1 in the first period and 2.2:1 in the second.

Overall survival was $33 \%$ and $43 \%$ for groups 1 and 2, respectively, with a trend towards better survival in the second period (not statistically significant) (Fig. 2). If absconding patients were excluded, event-free survival was $50 \%$ for group 1 and improved to $68 \%$ for group 2, not yet statistically significant but with a trend towards significance $(p=0.18) \quad$ (Fig. 3).
6 - 129). Overall survival for children with unilateral disease was $59 \%$ v. $62 \%$ for bilateral disease (combined data for groups 1 and 2, excluding absconders). As mentioned above, 13/34 patients with bilateral disease had metastasis at diagnosis (38\%) (Fig. 6).

The major reason for poor outcome was progression of disease ( 9 patients in group 1,11 in group 2), followed by relapse in 5 patients in each group (28\% and $22 \%$ for groups 1 and 2, respectively). Other reasons included sepsis in 4 patients (2 with limited and bilateral disease, 2 with metastatic disease), an underlying congenital heart lesion in 1 patient, underlying renal disease in 1 patient, and unexpected death of 1 patient at home. One of the patients with limited bilateral disease, who died of sepsis (Pseudomonas aeruginosa), also had concomitant HIV infection.

\section{Discussion}

Limited disease at diagnosis of $\mathrm{RB}$ offers the best chance of cure, with $>90 \%$ of children surviving in developed countries, and also ensures that these children qualify for conservative eye-sparing treatment with decreased morbidity, especially as it is possible to avoid external beam radiation therapy. ${ }^{[1]}$ Late diagnosis and advanced disease are common in developing countries, however, and associated with an increased risk of extraocular disease and poor outcome. ${ }^{[4,5]}$ Canturk et al. ${ }^{[14]}$ reported estimated survival for low-income countries as $40 \%$, for lower middle-income countries (LMICs) as $77 \%$ and for upper middle-income countries (UMICs) as 79\%. In our study, survival rates were $50 \%$ and $68 \%$, respectively, in the two time periods, excluding absconding patients, which is lower than the survival achieved in either LMICs or UMICs. Of concern is that about $25 \%$ of patients in each study period absconded during or after treatment, which lowered overall survival to $33 \%$ and $43 \%$, respectively, for the two time periods and contrasts with an Indian report for 2008 - 2011, where a statistically significant downward trend in the rate of absconders was seen. ${ }^{[15]}$ Kumar et al. ${ }^{[15]}$ further reported that the major reasons for abandonment of treatment were either financial constraints $(30 \%)$ or refusal of enucleation $(20 \%)$, which was probably also true for our study population.

The gender distribution of our patients was similar to that in developed countries, but the unilateral v. bilateral ratio was $73 \%$ v. $27 \%$. This ratio correlated with a previous SA study in the 1970s in which Freedman and Goldberg ${ }^{[16]}$ found that $82 \%$ of patients 
had unilateral disease, which was different from developed countries, which had a $60 \%$ v. $40 \%$ ratio. Age was higher for unilateral disease (35 months) than for bilateral disease (21 months), which has been reported previously in developing countries, but overall our patients were older than those in developed countries. ${ }^{[1,4]}$

Canturk et al ${ }^{[14]}$ further reported that the presence of metastatic disease was significantly associated with physician density. There is a general shortage of health professionals in SA state hospitals, and Hlangani ${ }^{[17]}$ reported in 2002 that more than 29000 doctors' posts were vacant throughout hospitals in SA. This may explain the large proportion of patients in this study with advanced disease at diagnosis ( $57 \%$ for the earlier and $40 \%$ for the later time period). Long distances from tertiary healthcare facilities that provide comprehensive therapy to children with $\mathrm{RB}$ are another factor that may result in late diagnosis. Access to POUs is available in eight centres in SA, but these centres are concentrated mainly in the large cities, whereas the majority of the patients in our study were from rural communities, far from these cities. The distances between cities and rural areas might have caused delays in the referral of these patients as a result of transport issues, added to late recognition of the cancer due to low physician density. ${ }^{[1,17]}$

There was improvement between the two study periods, with a trend towards more limited disease and better survival in the second period. Public healthcare interventions such as free healthcare for children under 6 years of age in SA and compulsory community service for doctors since 1998 did not seem to have influenced early diagnosis of childhood cancer significantly, which was contrary to our expectations. ${ }^{[7-9]}$

Early diagnosis campaigns are probably the solution. ${ }^{[18,19]}$ Parents should be taught to look for the presence of a red reflex of the eye by taking a flash photograph of their children younger than 1 year of age and to report the absence of this reflex, or the presence of any white spot in the eye, at the nearest primary care facility, which may improve early diagnosis. Healthcare workers also need training to increase their awareness that leucocoria in a young child is probably due to $\mathrm{RB}$, and refer these patients urgently to a tertiary healthcare facility for further management. In Honduras information about leucocoria was distributed at vaccination clinics, targeting mothers to recognise the symptoms to improve early diagnosis. ${ }^{[20]}$ Kenya launched a 5-year capacity-building national strategy to improve the survival of children with RB in 2008. ${ }^{[21]}$ Key components of the Kenyan National Retinoblastoma Strategy are efforts to create $\mathrm{RB}$ awareness among healthcare workers and the public at large, to improve diagnostic pathology services, and to implement a support programme for families with a child suffering from RB. A similar programme is needed in SA to assist in early recognition of RB by creating awareness, and ensure optimal care to cure the disease and save vision.

\section{Conclusion}

Advanced disease in this study was associated with an overall survival rate of $<70 \%$. A strategy is necessary in SA to ensure that children with RB are diagnosed early and that $>90 \%$ of them are cured. This will require mass education programmes directed at the public at large to sensitise them to the significance of leucocoria, as well as at healthcare workers to teach them to diagnose the disease early and refer patients timeously to the multidisciplinary teams at the existing POUs in SA, of which the majority initiated a standardised RB management protocol in January 2013.

Acknowledgements. We acknowledge the assistance of the Department of Radiation Oncology, Faculty of Health Sciences, University of Pretoria, for external beam radiotherapy, and Drs Clare Stannard and Karin Lecouna of the Departments of Radiation Oncology and Opthalmology, respectively, Groote Schuur Hospital and University of Cape Town, for iodine-125 brachytherapy.

\section{References}

1. Hurwitz RL, Shields CL, Shields JA, et al. Retinoblastoma. In: Pizzo PA, Poplack DG, eds. Principles and Practice of Pediatric Oncology. 6th ed. Philadelphia: Lippincott-Raven, 2011:809-837.

2. Theriault BL, Dimaras H, Gallie BL, et al. The genomic landscape of retinoblastoma: A review. Clin Exp Ophthalmol 2014;42(1):33-52. [http://dx.doi.org/10.1111/ceo.12132]

3. Stiller CA, Parkin DM. Geographic and ethnic variations in the incidence of childhood cancer. Br Med Bull 1996;52(4):682-703. [http://dx.doi.org/10.1093/oxfordjournals.bmb.a011577]

4. Chantada GL, Fandiňo A, Manzitti J, et al. Late diagnosis of retinoblastoma in a developing country. Arch Dis Child 1999;80(2):171-174. [http://dx.doi.org/10.1136/adc.80.2.171]

5. Boubacar T, Fatou S, Fousseyni T, et al. A 30-month prospective study on the treatment of retinoblastoma in the Gebriel Toure Teaching Hospital, Bamako, Mali. Br J Ophthalmol 2010;94(4):467-469. [http:// dx.doi.org/10.1136/bjo.2009.159699

6. Navo E, Teplisky D, Albero R, et al. Clinical presentation of retinoblastoma in a middle income country. J Pediatr Hematol Oncol 2012;34(3):97-101. [http://dx.doi.org/10.1097/MPH.0b013e31821d18f9]

7. McCoy D. Free Health Care for Pregnant Women and Children Under Six in South Africa. http:// www.hst.org.za/publications/free-health-care-pregnant-women-and-children-under-six-south-africa (accessed 13 January 2014)

8. Reid SJ. Compulsory community service for doctors in South Africa - an evaluation of the first year S Afr Med J 2001;91(4):329-336.

Nemutandani MS, Maluleke FRS, Rudolph MJ. Community service doctors in Limpopo Province. S Afr Med J 2006;96(3):180-182.

10. Poyiadjis, S, Wainwright L, Naidu G, et al. The Saint Siluan warning signs of cancer in children: Impact of education in rural South Africa. Pediatr Blood Cancer 2011;56(2):314-316. [http://dx.doi. org/10.1002/pbc.22853]

11. Grabowski EF, Abramson DH. Intraocular and extraocular retinoblastoma. Hematol Oncol Clin North Am 1987;1(4):721-735

12. Stannard C, Sealy R, Hering E, et al. Localized whole eye radiotherapy for retinoblastoma using a ${ }^{125}$ applicator, 'claws'. Int J Radiat Oncol Biol Phys 2001;51(2):399-409. [http://dx.doi.org/10.1016/S03603016(01)01638-8]

13. Stannard C, Sealy R, Hering E, et al. Postenucleation orbits in retinoblastoma: Treatment with ${ }^{125} \mathrm{I}$ brachytherapy. Int J Radiat Oncol Biol Phys 2002;54(5):1446-1454. [http://dx.doi.org/10.1016/S03603016(02)03756-2]

14. Canturk S, Qaddoumi I, Khetan V, et al. Survival of retinoblastoma in less-developed countries impact of socioeconomic and health-related indicators. Br J Ophthalmol 2010;94(11):1432-1436. [http:// dx.doi.org/10.1136/bjo.2009.168062]

15. Kumar A, Moulik NR, Mishra RK, et al. Causes, outcome and prevention of abandonment in Kumar A, Moulik NR, Mishra RK, et al. Causes, outcome and prevention of abandonment in
retinoblastoma in India. Pediatr Blood Cancer 2013;60(5):771-775. [http://dx.doi.org/10.1002/

16. Freedman J, Goldberg L. Incidence of retinoblastoma in the Bantu of South Africa. Br J Ophthalmol
16 1976;60(9):655-656

17. Hlangani M. Brain drain: Systems on life support. The Star 2002; 22 October. http://www.healthlink. org.za/news/20021030 (accessed 13 January 2014).

18. MacCarthy A, Birch JM, Draper GJ, et al. Retinoblastoma: Treatment and survival in Great Britain

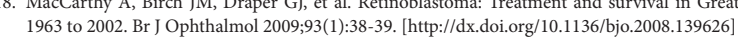

1963 to 2002. Br J Ophthalmol 2009;93(1):38-39. [http://dx.doi.org/10.1136/bjo.2008.139626]
Bowman RJ, Mafwiri M, Luthert P, et al. Outcome of retinoblastoma in East Africa. Pediatr Blood Cancer 2008;50(1):160-162. [http://dx.doi.org/10.1002/pbc.21080]

20. Leander C, Fu LC, Pena A, Howard SC, Rodriguez-Galindo C, et al. Impact of an education program . Leander C, Fu LC, Pena A, Howard SC, Rodriguez-Galindo C, et al. Impact of an education program
on late diagnosis of retinoblastoma in Honduras. Pediatr Blood Cancer 2007;49(6):817-819. [http:// on late diagnosis of retinobla

21. Dimaras H, White A, Gallie B. The Kenyan national retinoblastoma strategy: Building local capacity in the diagnosis and management of pediatric eye cancer in Kenya. Ophthalmology Rounds $2008 ; 6(4)$ www.ophthalmologyrounds.ca/crus/ophthcdneng0708_08.pdf (accessed 13 November 2013). 